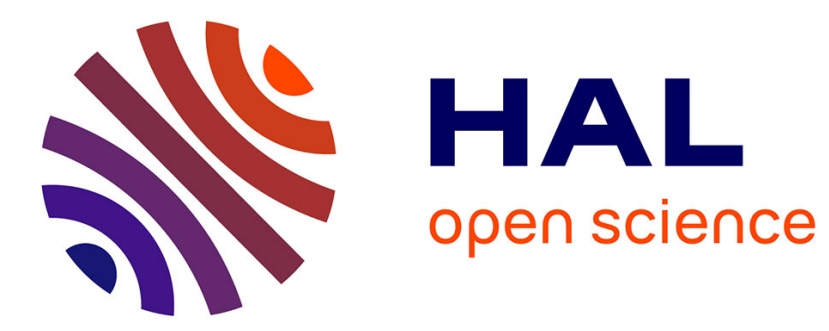

\title{
Robust Tracking of a Two-Fingered Micromanipulation System Working Through the Focus of an Optical Beam
}

Nabil Amari, David Folio, Antoine Ferreira

\section{To cite this version:}

Nabil Amari, David Folio, Antoine Ferreira. Robust Tracking of a Two-Fingered Micromanipulation System Working Through the Focus of an Optical Beam. American Control Conference (ACC'2014), Jun 2014, Portland, OR, USA, United States. pp.1613-1618, 10.1109/ACC.2014.6859244 . hal01070678

\section{HAL Id: hal-01070678 \\ https://hal.science/hal-01070678}

Submitted on 2 Oct 2014

HAL is a multi-disciplinary open access archive for the deposit and dissemination of scientific research documents, whether they are published or not. The documents may come from teaching and research institutions in France or abroad, or from public or private research centers.
L'archive ouverte pluridisciplinaire HAL, est destinée au dépôt et à la diffusion de documents scientifiques de niveau recherche, publiés ou non, émanant des établissements d'enseignement et de recherche français ou étrangers, des laboratoires publics ou privés. 


\title{
Robust Tracking of a Two-Fingered Micromanipulation System Working Through the Focus of an Optical Beam
}

\author{
Nabil Amari, David Folio and Antoine Ferreira
}

\begin{abstract}
This paper reports a control strategy of two AFM gripper for manipulation on analysis Beam, using dual micro/nano manipulators in order to grippe a micro spherical ball and tacking-maintain on the focus of laser beam. The main idea is to control and drive AFM gripper to expose micro samples in the laser beam by localization of the maximum intensity laser beam given by a four-quadrant photodiode. The focalization of analysis Beam used for microhandling is few micrometer that making the intensity measurement sensitive at the externals variations and positioning of the four-quadrant photodiode affecting the control of the gripper and position the sample far of the focalization of laser beam. To overcome this problem, the Particle Filter (PF) algorithm is used to estimate the position of laser beam.The dual manipulators is controlled by combine different performance dynamics (micro manipulator and nano manipulator) to track a laser beam with high precision.
\end{abstract}

\section{INTRODUCTION}

The Beam analysis(laser beam, synchrotron radiation) represent a great tool for comprehension physic phenomena with small focalization. In biology it is became possible to detects different pathologies with precision by micro cellular samples diagnostics in the infrared. The difficulty is how to maintain a micro sample in the focus of the laser beam that depends of the focal localization precision. The micro manipulation systems can answer for this request by nano gripper driven with high-precision positioner[]. The Fig.1 shows a protocol manipulation using two gripper to achieve sequential micro griping of micro sample and transport it to focus of laser Beam. In such a system involves dual manipulators system contain different dynamics and used piezoelectric-stack actuated nanomanipulators, various control design architectures and methodologies have been developed for dual-stage servo control designs to deal with uncertainties (modeling errors, sensor limitations) and environment noise. They can be largely classified into two categories: those based on decoupled or sequential single-inputsingle-output (SISO) designs, and those based on modern optimal design methodologies, such as LQG, LQG/LTR, Hinfinity, and -synthesis, in which the dual-stage controllers are obtained simultaneously[][].

To maintain the micro sample in the focus of laser beam, in addition to the handling system, also depends on the position of the laser beam a local search of the maximum laser beam position is needed to control the micro handling system,

N. Amari, D. Folio and A. Ferreira are with the Laboratoire PRISME EA 4229; Ecole Nationale Suprieure d'Ingnieurs de Bourges, 88 boulevard Lahitolle, 18020 Bourges, France. Corresponding author: Antoine Ferreira (Email:antoine.ferreira@ensi-bourges.fr, Tel: +33 24848 4079)

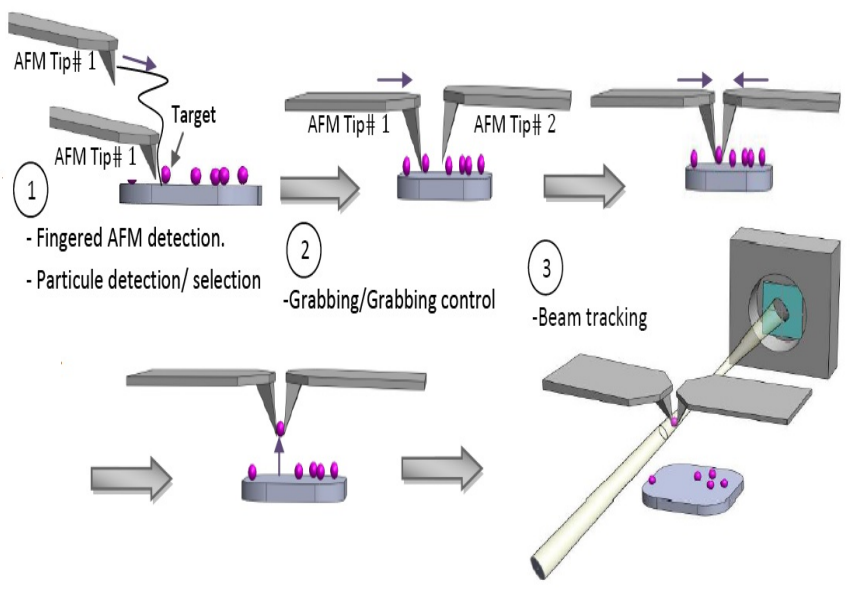

(a)

Fig. 1. High-precision position measurement based on laser beam transmission are commonly used in nanomanipulation applications.

usually the photodiode sensor device(PSD) is used for this task.

We design a control system that guaranteed microhandling tasks of micro samples in analysis beam. The purpose of this control system is to coordinate two AFM gripper mounted on a 6 dof dual-alignment system for maintain micro spherical ball on maximum laser beam intensity sensed by fixed four-quadrant PSD. The laser beam tracking processus by automated control a AFM gripper depends on real-time of laser beam maximum intensity positioning, where the Particle Filter (PF) algorithm is used to estimate the position of the laser beam in real-time, transmitted at each precision dual alignment system . A Control strategy is adopted to drive each dual alignment system composed by different dynamics by a single laser beam estimation feedback.

The paper is divided into five sections. Section 2 describes the experimental setup. Section 3 describes the dynamics which is involved in the control. Section 4 describes the different control step of a Two-Fingered. Section 5 presents experimental results for the performance of the beam laser beam tracking.

\section{EXPERIMENTAL SETUP}

The control scheme of the beam pointing and tracking is shown in Fig.2. composed of: i) Handling device comprises two fingered (Kleindiek FMT-400: $400 \mathrm{~m}$ force sensors) mounted in each compact computer-controllable XYZ alignment and positioning systems combining the advantages of ultra-high-resolution piezo drives(to $120 \times 120 \times 120 \mu \mathrm{m}$ ) 


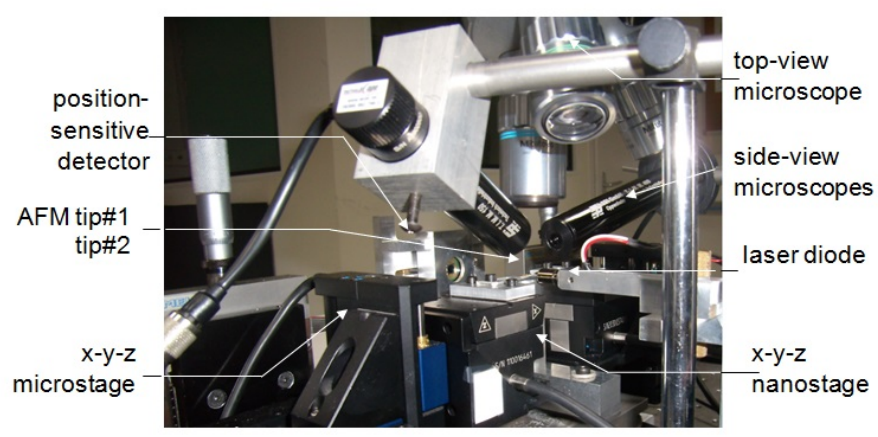

Fig. 2. Experimental setup.

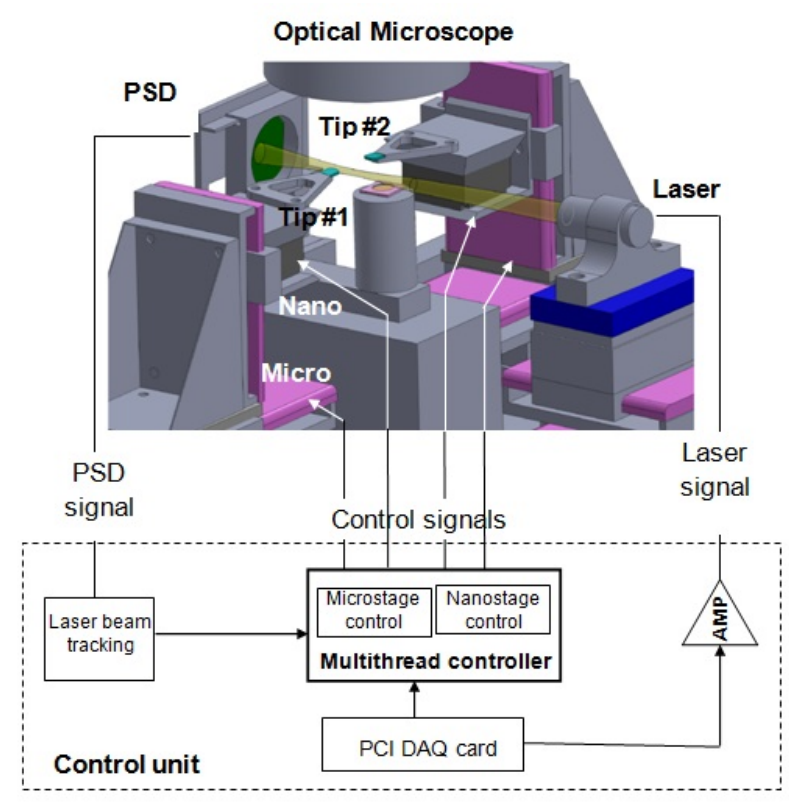

Fig. 3. Schematic diagram of the architecture of the laser beam tracking control system.

i.e., $x-y-z$ closed-loop nanostage (P-611.3S NanoCube from Physics Instruments, characterized by high bandwidth) with the long travel range of motorized stages (to $15 \times 15 \times 15$ $\mathrm{cm}$ ) i.e., X-Y-Z closed-loop microstage (F-130 DC from Physics Instruments). ii)Optical device placed in side view of the handling device, integrate a $635 \mathrm{~nm}$ laser diode(Red) Lightshow for producing laser beam, where the laser beam position is measured by the image laser beam formed on a four-quadrant position sensing device(PSD). iii)Imaging device for top-view (top optical microscope - Mituyo $\times 50$ ) and side-view (TIMM $\times 400)$ used for localization and guidance.

Fig.3 shows the overall control scheme for power, laser beam tracking and fingered control. The control strategy of two fingered for microrobotic manipulation tasks and maintain a spherical ball on maximum laser beam intensity by tracking laser beam motion (Brownian or stochastic trajectory) are processed in real-time using MATLABs xPC software. For laser beam intensity maximum detection and beam tracking a highspeed data acquisition (DAQ) (NI 6289) card is used to register photodiode voltage output, generated when the PSD is illuminated. A multi-thread structure is developed to independently control and coordination of fingered for parallel manipulation, object/Beam detection, handling and tracking laser Beam motion.

\section{DYNAMICS MODELING}

This section reviews the different model dynamics of the different system components.

\section{A. Dynamics of Piezoelectric and Magnetic Actuators}

The micro manipulation processus and the high precision laser beam tracking detected by PSD need a cooperative control of two fingered, i.e, control and combine the different dynamic of the micro/nano stage for drive each fingered in $x-y-z$ direction. For controller synthesis adopted in strategy control, and because we have no parametric information on drivers we need to set up dynamic modeling of dual micro/nano stages. The dynamic model of the Piezoelectric and Magnetic actuators are obtained by experiment estimated transfer functions based on responses of each actuator using random input signal. The dynamics models of the micro and nano are done by the following third-order approximation reduced order for each $x-y-z$ direction respectively :

$$
\begin{aligned}
& G_{\text {micro }(x, y, z)}(z)=\frac{b_{0}+b_{1} z^{-1}+b_{2} z^{-2}+b_{3} z^{-3}}{1+a_{1} z^{-1}+a_{2} z^{-2}+a_{3} z^{-3}} \\
& G_{n a n o(x, y, z)}(z)=\frac{b_{1} z^{-1}+b_{2} z^{-2}+b_{3} z^{-3}}{1+a_{1} z^{-1}+a_{2} z^{-2}+a_{3} z^{-3}}
\end{aligned}
$$

\section{B. Dynamics of Four Quadrant Detector and Laser Beam}

in our optical device the Photo Sensitive Detector(PSD) used consists of 4 photosensing parts separated by a small gap. Usually, The laser beam is pointed towards the dead center between the 4 quadrants and the beam diameter is selected to fit inside of the total quadrant area. When light falls on all four quadrants, they will generate current for each quadrant according to the light intensity and then amplified into voltage signals V1-V4 respectively.

The difference between the left and right quadrants $(\mathrm{Vx})$ and top and bottom quadrants $(\mathrm{Vy})$ can be used to indicate the offsets of the spot and be adjusted to zero by centering the beam, whereas the sum quadrants voltages corresponding at intensity laser beam (Vs) is at a maximum

$$
\begin{aligned}
& V_{x}=\left(V_{1}+V_{4}\right)-\left(V_{2}+V_{3}\right) \\
& V_{y}=\left(V_{1}+V_{2}\right)-\left(V_{3}+V_{4}\right) \\
& V_{s}=V_{1}+V_{2}+V_{3}+V_{4} .
\end{aligned}
$$

In Fig.4) that demonstrate the voltage signal $\mathrm{Vx}(\mathrm{Vy}$ is similar at $\mathrm{Vx}$ ) thereby become very sensitive to slight deviations in the position of the beam from this initial centered setting. Than $V_{x}$ and $V_{y}$ channel outputs are directly related to the energy of the laser beam that falls in each quadrant while $V_{s}$ is the sum voltage. It is assume that the light intensity on the laser's beam cross section obeys Gaussian distribution. The current generated by each sensing element can be described as given in: 


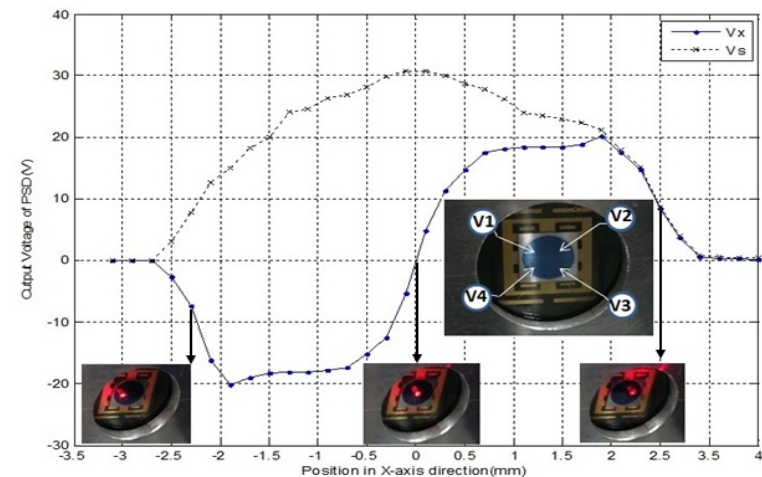

Fig. 4. Output voltage curve $V_{x}$ with a zoom in the block area near zero on an four-quadrant PSD.

$$
I=k_{1} \iint \frac{2 E_{l}}{\pi^{2} r} e^{\frac{2\left(x_{1}^{2}+y_{1}^{2}\right)}{r^{2}}} d x_{1} d y_{1}
$$

where $I$ is the current, $r$ the radius of the laser light spot, $E_{t}$ is the energy of the laser beam, $\left(x_{1}, y_{1}\right)$ is the coordinate of a point on the light spot in a coordinates system located at the center of the light spot, and $k_{1}$ is a coefficient. As shown in Fig.4)

- AFM, The Vs can be used to measure changes in the beam intensity, so this can be used to correct the $\mathrm{X}$ and $\mathrm{Y}$ output values for voltage changes that are due to intensity fluctuations rather than actual beam deviations by filtering.
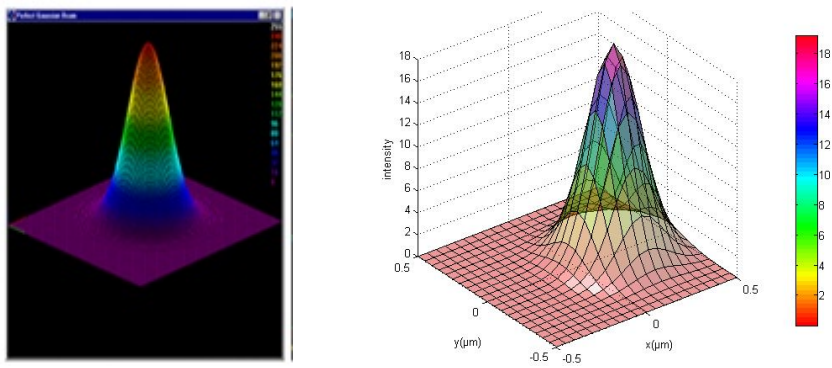

Fig. 5. Light intensity on the laser's beam cross section: (left) theoretical and (right) experimental intensity obeying to Gaussian distribution.

As we can see in Fig.5, the experimental intensity sensed by the PSD can be fitted with a gaussian distribution as calculated by the theoretical equation (4).

The maximum Gaussian intensity variation by the motion of the laser beam in $\mathrm{x}$ direction showing the position of the beam on the dial can be represented by Brownien seconde order variation given by:

$$
\frac{d^{2} x(t)}{d t^{2}}+\beta_{x} \frac{d x(t)}{d t}=W_{x}
$$

were $\beta_{x}$ coefficient of friction and $W_{x}$ is zero-mean Gaussian random variable with variance $\delta_{x}^{2}$. The $y$-axis can be modeled in the same manner as the $x$-axis, though with different dynamics. The discrete state space of eq5 laser beam is represented by:

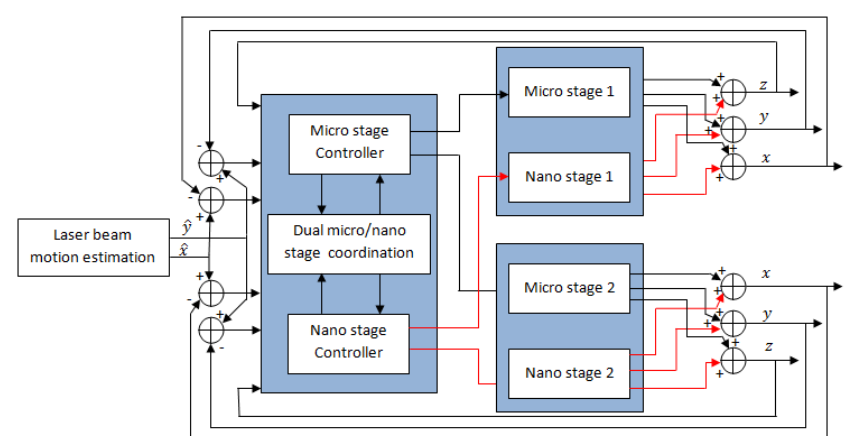

Fig. 6. Master-slaver controller with decoupling structure for maximum light tracking.

$$
\begin{gathered}
X_{k}=A X_{k-1}+B W_{k} \\
Y_{k}=C X_{k-1}
\end{gathered}
$$

where

$$
\left[\begin{array}{llll}
x_{k} & y_{k} & \dot{x}_{k} & \dot{y}_{k}
\end{array}\right]^{T}
$$

$\left(x_{k}, y_{k}\right)$ and $\left(\dot{x}_{k}, \dot{y}_{k}\right)$ are the source potion in the plane $x-y$ and velocity respectively.

\section{Control Scheme of A Two-Fingered AND LASER BEAM TRACKING}

The focusing of micro spherical ball handled by TwoFingered on maximum intensity laser beam and maintain it for several minutes without fingered loose the micro spherical ball during beam tracking, requires a robust control motion of each dual micro/nano manipulator(characterized by two independent control output micro controller and nano controller) and friability localization of laser beam position. Figure 7 presents the control scheme. The detection and estimation of the position of maximum laser beam intensity to drive fingered implies to anticipates the intensity variation caused by the dynamic of the beam laser by estimationprediction filters, transmitted for each dual micro/nano manipulator. The coordination the separated performance of the micro stage and nano stage are important to tack laser beam by only position feedback signal available and maintain the micro sphere ball.

\section{A. Particle filter approach}

The first step is to guarantied a real time maximum intensity position estimation sensitive at the laser beam motion. The particle filter is used for this Tasks, that implements the Bayesian filter by modeling the tracking problem as estimating the posterior distribution using the importance sampling of Monte Carlo methods, which approximates the real distribution by generating a set $\mathrm{N}$ samples $x_{k}^{i}$ distributed according to the posterior distribution of the Markov state process given the observations, $p\left(x^{t} \mid y^{t}\right)$, and their associate a normalized importance weights. The estimates calculation of 
the posterior distribution of the whole process by importance sampling method give:

$$
\begin{gathered}
p\left(x_{k} \mid y_{1: k}\right) \approx \sum_{i=1}^{N} w_{k}^{i} \delta\left(x_{k}-x_{k}^{i}\right) \\
w_{k}^{i} \propto w_{k-1}^{i} p\left(y_{k} \mid x_{k}^{(i)}\right)
\end{gathered}
$$

were normalized weights $\tilde{w}_{k}^{(i)}=w_{k}^{(i)} / \sum_{j=1}^{N} w_{k}^{(j)} i=1 \ldots . . N$. The particle filter is generally called the Sequential Importance Sampling (SIS) algorithm, which is limited by the degeneracy phenomenon [3]. The Sampling Importance Resampling (SIR) By adaptive multinomial resampling based on bootstrap method [4] is used to estimate and predicate laser beam positions and overcome the degeneracy problem of the original Particle Filter. At every $k_{t h}$ step each of the particles is propagated according to dynamical model as the importance distribution $p\left(x_{k}^{i} \mid y_{1: k}\right)=p\left(x_{K}^{i} \mid x_{K-1}^{i}\right)$, can be generated by first generating a process noise sample $v_{k-1}^{i} \sim$ $p_{v}\left(v_{k-1}\right.$ and setting $x_{k}^{i}=f\left(x_{k-1}^{i}, v_{k-1}^{i}\right)$. The particles are then resampled according to their new importance weights to ensure a uniform weight distribution i.e. $x_{k-1}^{i}=1 / N$ and so $w_{k}^{i} \propto p\left(y_{k} \mid x_{k}^{(i)}\right)$. Resample cycle is as follow : $\mathrm{N}$ sorted random numbers uniformly distributed in $[0,1]$ are selected. From the cumulative sums of the particle weights calculated, the number of the sorted random numbers that appear in each interval of the cumulative sum represents the number of copiers of this particular particle which are going to be propagated to the next generation. Intuitively, if a particle has a large weight then many random numbers are going to survive.

SIR particle filter approach:

- Generates $\mathrm{N}$ samples $\left\{x_{0}^{i}\right\}_{i=1}^{N}$ form the initial distribution

- Calculate the weights $w_{k}^{i} \alpha p\left(y_{k} \mid x_{k}^{i}\right), i=1, \ldots ., N$ and normalize $\tilde{w}_{k}^{(i)}=w_{k}^{(i)} / \sum_{j=1}^{N} w_{k}^{(j)}$.

- Generate a new set $\left\{x_{k}^{i *}\right\}_{i=1}^{N}$ by performing resampling.

- Predict new particles, $x_{k+1}^{i}=f\left(x_{k}^{i *}, v_{k}\right)$ ie using different noise realization for the particles.

- Compute the output of the SIR filter by: $\hat{x}_{k}^{N}=\sum_{i=1}^{N} w_{k}^{i} x_{k}^{i}$

In order to evaluate the performance of Particle Filter used to estimate position of laser beam we give to the laser beam a synthetic trajectory generated randomly without working zone. For particle filtering the particle number needed is determined so that the computational remained suitable for real time application, and assuring high performances, we have done several experiments to determine the appropriate number of particles used in the estimation step. The number of the particles ,thus choused $\mathrm{N}=80$.

The results of laser beam motion prediction using Particle Filter are presented in fig.7. At first glance, the filter succeed to fellow the true trajectories very closely. Another performances test are realized by comparing performance with a classical filter changing the velocity actuator of the laser beam motion and maintaining the initial parameters of the filter. the curve of the square error $\varepsilon(n)$ is represented in figure.8. As can be illustrate the performances of the particle filter are better that those of the classical filter in terms of precision were particle filter converge to the real position of the laser beam with a minimal error. As can be seen in fig.9 the particle filter commits less error than classical filter.

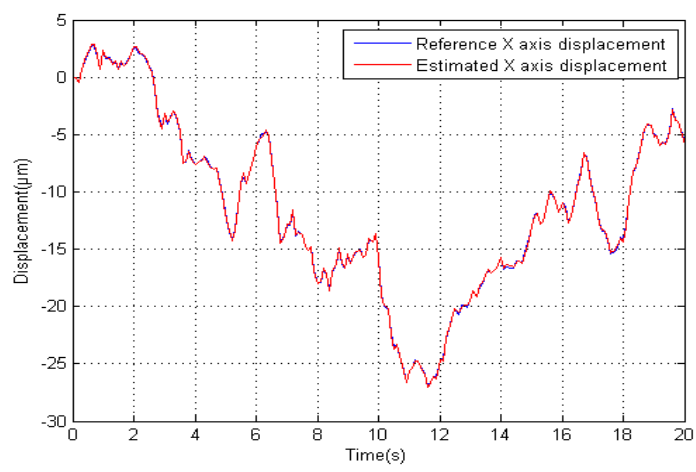

(a)

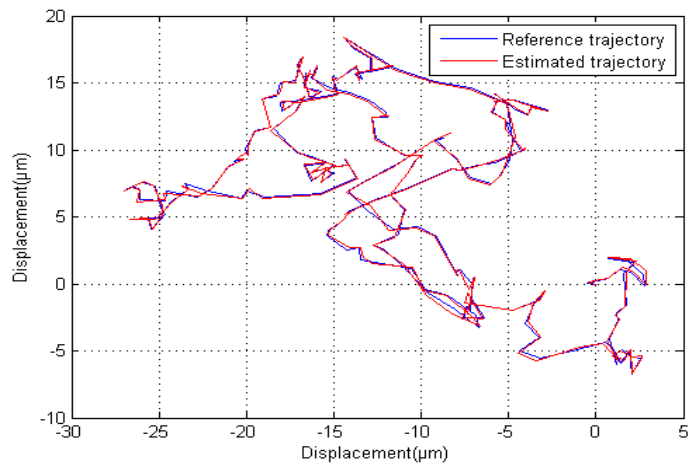

(b)

Fig. 7. Position estimation with particule filter: (a) Motion estimation on $x$ direction, (b) 2D motion

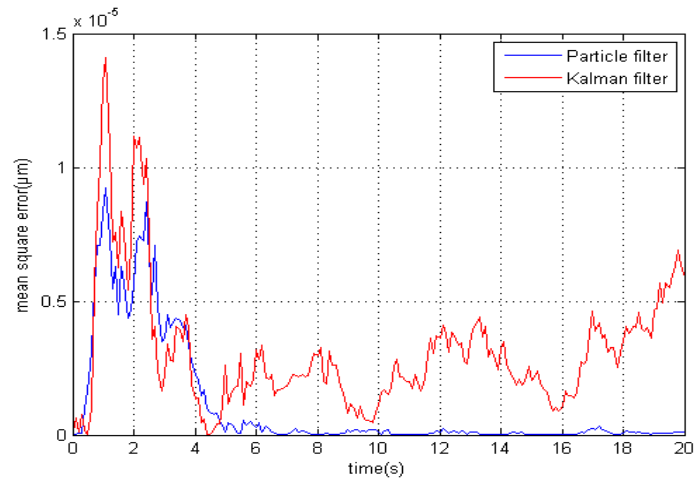

Fig. 8. Mean quadratic error

\section{B. $\mathrm{H}_{\infty}$ Controller}

Contrary to micro manipulator were PID controller is sufficient, a robust control strategies for piezoelectric 


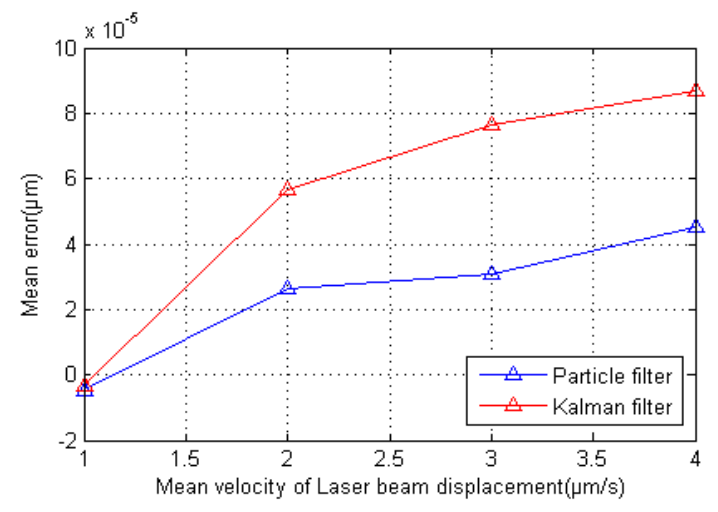

Fig. 9. mean quadratic error

actuated nanomanipulators is implemented to deal with uncertainties(modeling errors,sensor limitation,non lineaire effect) and environment noise.

The MIMO $H_{\infty}$ standard problem is used, because it have been successfully used in a wide variety of applications and it proved its efficiency in coping with model uncertainty. The $H_{\infty}$ concept is to represent system with the block diagram shown in fig.10 where $W_{i}$ are a weighting function. The $H_{\infty}$ standard problem consists to find a controller $K$ which stabilizes system and determine a positive number $\gamma_{0}$ to satisfy:

$$
\|T(s)\|_{\infty}<\gamma\left(\gamma \geq \gamma_{0}\right)
$$

Where $T(s)$ closed loop transfer function of the system represented in fig.10, given by:

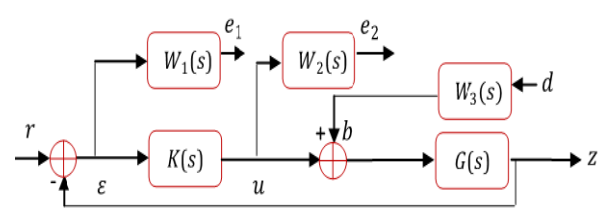

Fig. 10. $H_{\infty}$ structure

$$
T_{z w}=\left(\begin{array}{ll}
W_{1} S & W_{1} S G W_{3} \\
W_{2} K S & W_{2} K S G W_{3}
\end{array}\right)
$$

$S$ is the sensivity function $S=(I+G(s) K(s))^{-1}$. For Solving $H_{\infty}$ standard problem and obtain a good controller depends on appropriate choice of a structure weighting function with optimal parameters which reflect the robust stability and performance requirements. Since the axis coupling of the fine stage is negligible and the the 3 axis of nano stage have different dynamics, which lets us to choose:

$$
W_{i}=\operatorname{diag}\left(w_{i 1}, w_{i 2}, w_{i 3}\right) \quad \text { with } i=1: 3
$$

and

$$
\mathrm{w}_{1 i}=\frac{a_{1 i} p+a_{2 i}}{p+b_{1 i}} \quad \mathrm{w}_{3 i}=\frac{p+a_{3 i}}{b_{2 i} p+b_{3 i}} \quad \mathrm{w}_{2 i}=\text { const }_{i}
$$

The inverse of $w_{1 i}$ and $w_{3 i}$ are an upper bound on the desired sensitivity loop shape and complementary sensitivity function $T(s)$, the inverse $w_{2 i}$ will effectively limit the controller $u$ [5].

\section{Parameters optimization}

The GA approach [6] is used to compute the parameters weighting function namely: $a_{1 i}, a_{2 i}, a_{3 i}, b_{1 i}, b_{2 i}, b_{3 i}$ and const $_{i}$, and to obtain a optimal $H_{\infty}$ controller. The concept is to generate a values population of chromosomes representing the parameters to optimize, and subjected to check whether it satisfies the performance index or not. If any the chromosomes does not satisfy the performance index a new chromosome is generated repeatedly until satisfied. Assuming the parameters weighting function to be a binary bits of chromosomes $X_{k}$. Each of parameters is encoded using $N_{b}$ binary bits, and all the binary bits of these parameters are cascaded to generate a binary chromosome of length $N^{*} N_{b}$, were $N$ is the number of target parameters considered for optimization and $N_{b}$ necessary number of bits for one parameter binary coding. A population of $N_{p}$ chromosomes can be represented as:

$$
X_{k}=\left[x_{0}^{k} x_{1}^{k} \ldots x_{j}^{k}\right] 0 \leq k \leq N_{p}-1,0 \leq j \leq N-1
$$

The $x_{j}^{k}$ is the $j^{t h}$ coded parameter in the $k^{t h}$ chromosome. The chromosome will be constituted with the cascaded parameters weighting function for each axis. However, the chromosome of parameters weighting is generated within their corresponding minimum and maximum intervals. As $W_{1}$ should be low-pass filter and $W_{3}$ should be high-pass filter the parameters for optimization will have the same maximum and minimum limitation i.e:

$$
\begin{gathered}
x_{0,7,14}^{k} \epsilon\left[\begin{array}{ll}
a_{11}^{\min } & a_{11}^{\max }
\end{array}\right], x_{1,8,15}^{k} \epsilon\left[\begin{array}{ll}
a_{21}^{\min } & a_{21}^{\max }
\end{array}\right], \\
x_{2,9,16}^{k} \epsilon\left[\begin{array}{ll}
a_{31}^{\text {min }} & a_{31}^{\max }
\end{array}\right], x_{3,10,17}^{k} \epsilon\left[\begin{array}{ll}
b_{11}^{\text {min }} & a_{11}^{\max }
\end{array}\right], \\
x_{4,11,18}^{k} \epsilon\left[\begin{array}{ll}
b_{21}^{\text {min }} & b_{21}^{\max }
\end{array}\right], x_{5,12,19}^{k} \epsilon\left[\begin{array}{ll}
b_{31}^{\min } & a_{31}^{\max }
\end{array}\right], \\
x_{6,13,20}^{k} \epsilon\left[\text { const }_{1}^{\text {min }} \text { const }_{1}^{\text {max }}\right]
\end{gathered}
$$

After the encoding of the chromosomes has been developed, the controller synthesize is realized with the following computing procedure form :

Initialization:

- $a_{1 i} \epsilon\left[\begin{array}{ll}0 & 1\end{array}\right], a_{2 i} \epsilon\left[\begin{array}{ll}10 & 100\end{array}\right], a_{3 i} \epsilon\left[\begin{array}{ll}0 & 1\end{array}\right], b_{1 i} \epsilon\left[\begin{array}{lll}0.001 & 0.01\end{array}\right]$ $b_{2 i} \epsilon\left[\begin{array}{ll}0 & 1\end{array}\right], b_{3 i} \epsilon\left[\begin{array}{ll}0 & 1\end{array}\right]$, const $_{i} \epsilon\left[\begin{array}{lll}0.01 & 1\end{array}\right]$

- Number of parameters $N=21$.

- Number of generations $G_{n}=100$.

- Bits number $N_{b}=5$.

- Chromosomes number $N_{p}=7$.

- Population size $P_{z}=\left(N_{p}, N * N_{b}\right)$.

- Crossover rates $=0.75$ and mutation rates $=0.3$.

1- Generate randomly the initial binary population of weights parameters.

2- Repeat for each generation :

2.1- Convert binary population to a decimal population by quantum :

$$
q(r)=[\max (r)-\min (r)] / 2^{N-1} 1 \leq r \leq N
$$


2.2- Evaluate the individual fitness function of all individuals by $f_{i}=\|T(s)\|_{\infty}\left(i=1, N_{p}\right)$.

2.3- Apply the Elitism selection technique to select the best-fit individuals for reproduction:

- compute the total fitness: $F=\sum_{i=1}^{N_{p}} f_{i}$.

- compute the means fitness: $F_{m}=\frac{f_{i}}{F}$.

- for each chromosome, compute $: e_{j}=\frac{f_{j}}{F_{m}}$ (where $f_{i}=$ array of fitness function of chromosomes) the chromosome $\mathrm{i}$ is reproduced integer part of $e_{j}$.

2.4- Breed new individuals through crossover and aleatory mutation operations of genes to obtain new chromosomes.

2.5- Replace least-fit population with new individuals.

3- After obtain optimal weights, use Riccati approach to synthesize $H_{\infty}$ controller.

4- Use a reduction method based on singular values to reduce the controller order .

For displacement on $\mathrm{x}$ axis direction the computing procedure allows to obtain the following weighting function:

$$
W_{11}=\frac{s+1}{s+0.9258} \quad W_{31}=\frac{s+0.005935}{0.913 s+0.9032} \quad W_{21}=0.7097
$$

The optimized discrete transfer function $K(z)$ is given by:

$$
K_{x}(z)=\frac{1.186 z^{4}-4.01 z^{3}+5.623 z^{2}-3.953 z+1.154}{z^{4}-3.25 z^{3}+4.405 z^{2}-3.06 z+0.9044}
$$

then,we obtain $u_{x}(k)$.

and corresponding $H_{\infty}$ norm $=1.010$. The fig.11 represents the fitness evolution for each chromosome of the population by generation, as can be seen from fig.11(a) a rapid convergence of the fitness.the chromosome population having a maximum fitness for each generation are selected and represented in the fig.11(b) as can be seen again the convergence of fitness to a optimal value $\gamma_{o p t}=1.01$, which assumes many solution of parameters are envisaged.As GA exhibits an excellent characteristic of global search and selection technic, the chromosomes optimal converge to a same optimal value.For illustrates the parameters optimization convergence, the fig. 12 represents the $W_{31}$ parameters optimization evolution.

\section{EXPERIMENTS}

The control strategy exposed in section 4 to control each AFM tip piloted by dual micro/nano actuators is implemented in our experimental platform. Fig.13, shows the microhandling task of a 20um spherical in order to focus on the laser beam. First we positioned the laser beam in sensed region of the four quadrant photodiode. The localization and position estimation of the laser beam intensity sensed by the four quadrants photodiode realized by the particle filter allowed a maximum local search by each AFM tips to actuate manipulation and positioned spherical ball in the focus of the laser beam, the Fig13(a) shows the detection of one AFM tip by the laser beam that demonstrate the robustness of particle filter to determinate a localization of maximum intensity and ensure to maintain micro sample in the focus of laser if the position of laser beam change. In the

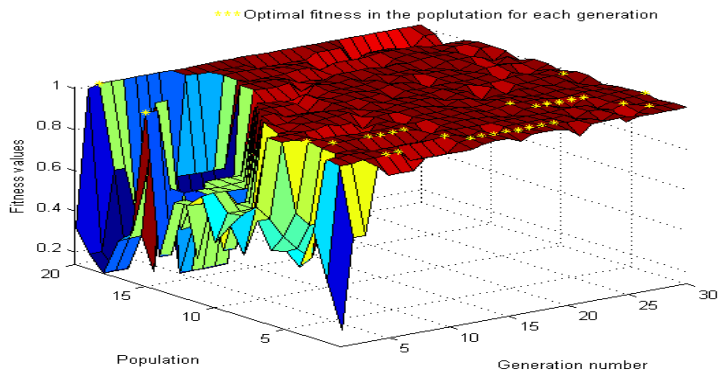

(a)

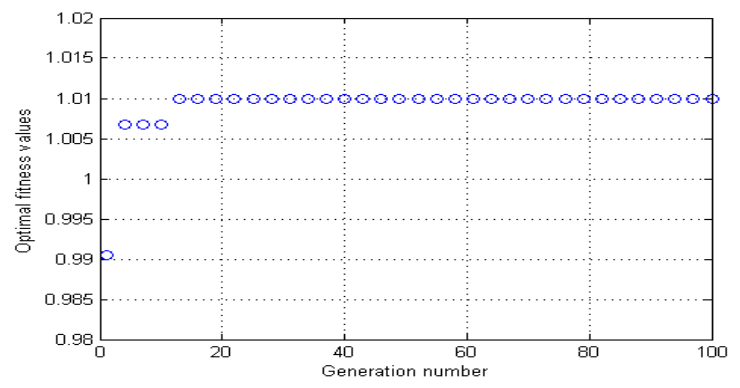

(b)

Fig. 11. Fitness evolution: (a): Population fitness computed for each generation, (b): Optimal fitness
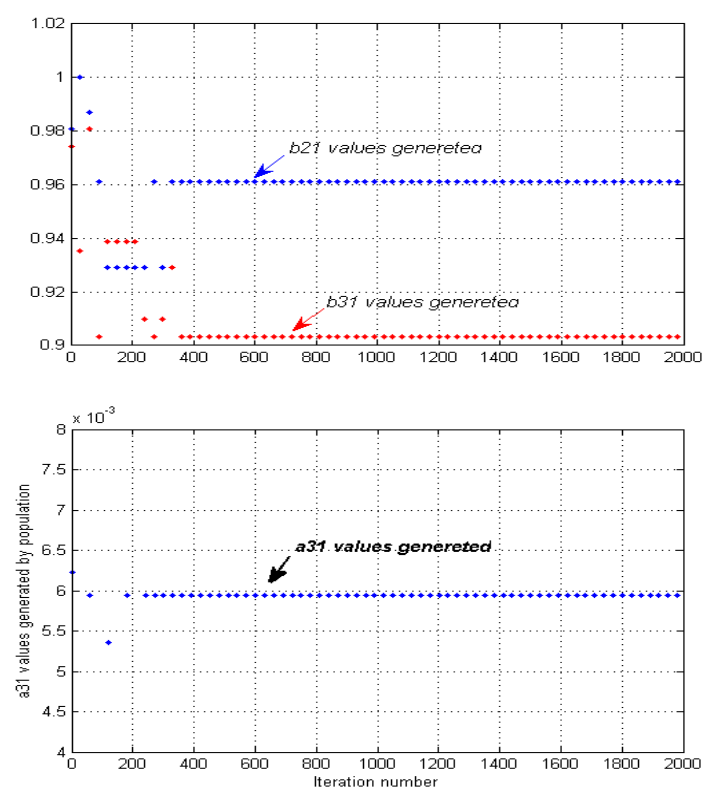

Fig. 12. $w_{31}$ parameters evolution for $x$ axis displacement

second step The gripping and the transport of the sample is realized successfully by approaching each tip close of the target(Fig.13(b)) and demonstrate the control precision of each dual micro/nano actuators. The AFM tips cooperation to grippe and move micro spherical (Fig.13(c)) shows the robustness of strategy control used for AFM tip's. 

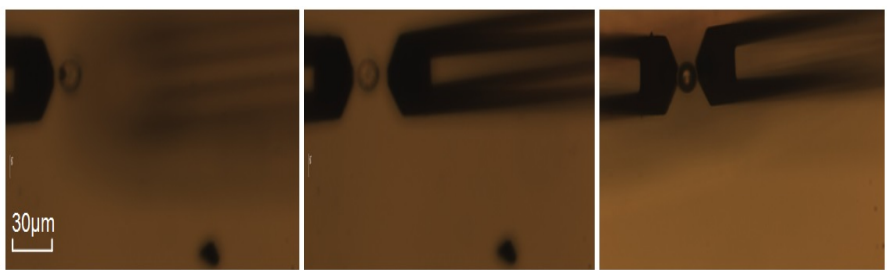

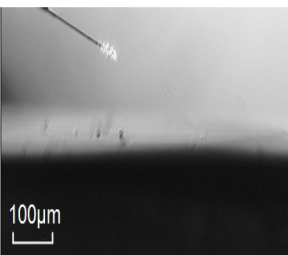

(a)

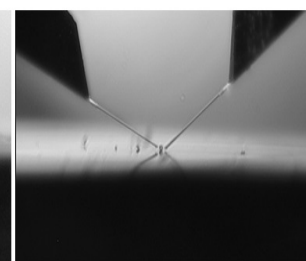

(b)

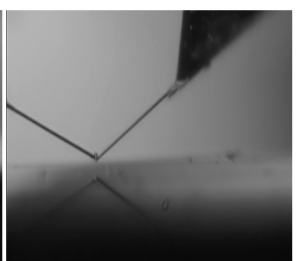

(c)

Fig. 13. Microhandling task of a $20 \mu \mathrm{m}$ spherical bead using a dualnanomanipulation system: (a) detection of the AFM tip1 by the laser beam, (b) approach of the AFM tip1 and AFM tip2 close to the microobject and (c) stable and reliable handling of the microobject.

\section{CONCLUSIONS \\ REFERENCES}

[1] A. Ehrlicher, T. Betz, B. Stuhrmann, D. Koch, V. Milner, M. G. Raizen, and J. Ks, Proc. Natl. Acad. Sci. U.S.A. 99, 16024 (2002)

[2] B. Stuhrmann, M. Ggler, T. Betz, A. Ehrlicher, D. Koch, and J. Ks, Automated tracking and laser micromanipulation of motile cells, Rev. Sci. Instrum. 76, 035105 (2005)

[3] H. Xie, S. Rgnier, High-Efficiency Automated Nanomanipulation with Parallel Imaging/Manipulation Force Microscopy, IEEE Transactions on Nanotechnology, Vol.11, Iss.1, pp:21-33, 2012.

[4] Pawel K. Orzechowski, James S. Gibson, Tsu-Chin Tsao, Optimal Disturbance Rejection by LTI Feedback Control in a Laser Beam Steering System, 43rd IEEE Conference on Decision and Control, December 14-17, 2004, Atlantis, Paradise Island, Bahamas, pp. 21432148.

[5] C.C. Lee, J. Park, Temperature Measurement of Visible Light-Emitting Diodes Using Nematic Liquid Crystal Thermography With Laser Illumination, IEEE Photonics Technology Letters, Vol. 16, NO. 7, July 2004.

[6] R. B. Evans, J.S. Griesbach, W.C. Messner, Piezoelectric microactuator for dual-stage control, IEEE Transactions on Magnetics, Vol.35, pp.977-981,1999.

[7] Nstor O. Prez-Arancibia, James S. Gibson, and Tsu-Chin Tsao, Observer-Based Intensity-Feedback Control for Laser Beam Pointing and Tracking, IEEE Transactions on Control Systems Technology, Vol.20, No.1, 2012, pp.31-47.

[8] Tran Trung Nguyen, A. Amthor and C. Ament, High Precision Laser Tracker System for Contactless Position Measurement, 2011 IEEE International Conference on Control System, Computing and Engineering, pp.97-102. 